\title{
Deficiency of prostacyclin production in meningococcal shock
}

\author{
R S Heyderman, N J Klein, G I Shennan, M Levin
}

\begin{abstract}
A deficiency of prostacyclin (PGI ${ }_{2}$ ) production by the vascular endothelium might underline the severe vasoconstriction and intravascular thrombosis that characterise meningococcal shock. The effect on $\mathbf{P G I}_{2}$ synthesis by human umbilical vein endothelial cells (HUVEC) in culture was examined in sera from children with meningococcal shock, healthy adults, and children with other febrile illnesses. In comparision with adult controls, $\mathbf{P G I}_{2}$ synthesis was reduced when HUVEC were incubated with the sera from 10 of 13 patients with meningococcal shock. A similar defect was observed with only four of 20 sera from children with other febrile illnesses. The effect of sera from patients with meningococcal shock on HUVEC was reversible with normal serum, and seems to be due to the absence of a factor necessary for $\mathbf{P G I}_{2}$ production rather than an inhibitor.

These findings suggest that a deficiency of $\mathbf{P G I}_{2}$ may have a role in the pathogenesis of meningococcal shock and that exogenous $\mathbf{P G I}_{2}$ may be of therapeutic benefit.
\end{abstract}

Despite early diagnosis and appropriate treatment the mortality from meningococcal infection has not decreased over the last 30 years. The case mortality overall is $10 \%$ and rises to an unacceptable $40 \%$ for those with septicaemia and shock. ${ }^{1}$ Attempts to improve the prognosis have so far been hampered by a lack of understanding of the pathophysiology of the disorder.

Clinical observation suggests that there are three major processes involved in the pathogenesis of meningococcal shock. Firstly, an increase in vascular permeability allows albumin to leak from the circulation, resulting in profound hypovolaemia and oedema. ${ }^{2}$ Secondly, intense vasoconstriction of some vascular beds and dilatation of others leads to impaired organ perfusion. ${ }^{3}$ Thirdly, intravascular thrombosis of both large and small vessels associated with platelet and clotting factor consumption intensifies tissue ischaemia. ${ }^{4}$ These three features suggest that in meningococcal sepsis there is disruption of the homoeostatic function of the endothelium that normally maintains vascular permeability and thromboresistance.

Disseminated intravascular coagulation, with activation of clotting factors and deposition of fibrin within the microvasculature, has been considered to be an essential component of meningococcal disease. Several studies have documented both thrombocytopenia and derangement of coagulation. ${ }^{45}$ However, the mechanism responsible for the thrombocytopenia and the role of the disordered endothelialplatelet interactions in the pathophysiology of meningococcal infection have not been well studied.

Prostacyclin $\left(\mathrm{PGI}_{2}\right)$ is a potent inhibitor of platelet aggregation and a powerful vasodilator. ${ }^{6}$ It is released from membrane bound arachidonic acid and is actively produced by the vascular endothelium. Together with other important endothelium derived platelet inhibitory factors, such as nitric oxide, ${ }^{7} \mathrm{PGI}_{2}$ has an important role in thromboresistance. $\mathrm{PGI}_{2}$ production increases appreciably in response to hypoxia and stress, ${ }^{8}$ suggesting that under these circumstances it may be particularly important in preventing intravascular platelet activation and tissue ischaemia.

We postulated that a deficiency of $\mathrm{PGI}_{2}$ synthesis by the endothelium might be involved in the intravascular thrombosis and vasoconstriction seen in meningococcal disease. We suggest that this defect may be due to the absence of a factor necessary for $\mathrm{PGI}_{2}$ production or the presence of an inhibitor in the serum of patients with the disorder. We therefore investigated the effect of sera from patients with meningococcal shock on endothelial $\mathrm{PGI}_{2}$ production, and have compared this with that seen from sera from children with other febrile illnesses.

\section{Patients and methods}

PATIENTS WITH MENINGOCOCCAL DISEASE

Twenty three children (mean age 4.6 years; range 0.3 to 12.7 years) with severe meningococcal disease, referred to the Hospital for Sick Children, Great Ormond Street over the last six years, were studied. The diagnosis was made clinically on the basis of a characteristic purpuric rash in all cases and was confirmed by culture and/or serum antigen test in 18 patients. The haemophilus rapid antigen test was negative in the remaining patients. Shock was detected in 18 patients, this was defined by at least two of the following: impaired peripheral perfusion, as signified by poor capillary return, oliguria (less than $1 \mathrm{ml} / \mathrm{kg} /$ hour) or hypotension (at least $2 \mathrm{SD}$ below the mean for age). Serum was available, in the acute phase, from only 13 of the patients with meningococcal shock.

\section{CONTROLS}

(a) Healthy adults

Serum was collected from 12 hospital workers 
who did not have a history of significant disease. This department has previously compared normal childhood and adult sera in the bioassay employed in this study and has not found any difference between the groups. Adult sera were therefore used to standardise the assay because of the ethical and logistic difficulties involved in obtaining samples from normal children.

\section{(b) Children with other febrile illnesses}

Serum was obtained from 20 children admitted to this hospital. Seven had non-meningococcal meningitis or encephalitis, one septicaemia, two Kawasaki disease, four toxic shock syndrome, two polyarteritis, and four had mild febrile illnesses. Shock, as defined by the criteria above, was diagnosed in six of these patients: four with toxic shock syndrome, one with haemophilus meningitis, and one with bacteroides septicaemia.

COLLECTION OF SAMPLES

Blood was collected within 24 hours of admission and serum separated within three hours. The samples were either used immediately or stored at $-70^{\circ} \mathrm{C}$ until required.

\section{CELL CULTURE}

Human umbilical vein endothelial cells (HUVEC) were isolated by established methods ${ }^{9}$ and cultured in Dulbecco's modified Eagle's medium containing $3.5 \mathrm{~g} / \mathrm{l}$ sodium bicarbonate to which $1 \%$ L-glutamine, $20 \%$ heat inactivated fetal calf serum, and $1 \%$ penicillin and streptomycin were added. Cells in first passage were grown to confluence in 24 well, flat bottom plates (Flow; $2 \cdot 0 \mathrm{~cm}^{2}$ per well).

\section{BIOASSAY FOR PGI 2 PRODUCTION BY HUVEC IN} CULTURE

Previously described methods were adapted to detect $\mathrm{PGI}_{2}$ in the supernatant of HUVEC in culture. ${ }^{10}$ All experiments were performed in duplicate. Twenty four well plates with confluent HUVEC were maintained at $37^{\circ} \mathrm{C}$ in a heating block. The wells were aspirated to near dryness and then incubated, in duplicate, with $500 \mu \mathrm{l}$ of culture medium (without fetal calf serum) containing $20 \%$ patient or control serum. After 10 minutes, $100 \mu$ l of culture supernatant was aspirated and added to $100 \mu \mathrm{l}$ of normal platelet rich plasma (platelet count 300 to $\left.500 \times 10^{9} / 1\right)$. The mixture was stirred for one minute, in a PAP-4 (BIO/Data) aggregometer, the platelets were then challenged with ADP $(2-4 \mu \mathrm{mol} / \mathrm{l})$, and aggregation recorded. The inhibition of platelet aggregation by the culture supernatants was expressed as a percentage of the maximal aggregation obtained with platelet rich plasma and fresh medium that had not been incubated with HUVEC (percentage full aggregation). Neither patient nor control serum was found to affect platelet aggregation without prior incubation with HUVEC, excluding the possibility that the sera used contained a factor or factors that stimulate platelet aggregation.

Where supernatants were found not to inhibit platelet aggregation, crossover experiments were performed. The culture supernatants used for the initial assay were removed and the cells were reincubated with fresh medium containing $20 \%$ control serum for 10 minutes. The culture supernatants were then assayed for recovery of $\mathrm{PGI}_{2}$ activity.

MEASUREMENT OF 6-KETO PGF $1 \alpha$

In order to confirm the results of the bioassay, the stable metabolite 6-keto $\mathrm{PGF}_{1 \alpha}$ was measured using a radioimmunoassay kit (Amersham). 6-Keto $\mathrm{PGF}_{1 \alpha}$ was measured in culture supernatants after incubation of HUVEC with the sera from seven children with meningococcal shock, which had been found not to induce $\mathrm{PGI}_{2}$ production employing the bioassay method and eight normal adult controls.

\section{STATISTICS}

For the $\mathrm{PGI}_{2}$ bioassay, a median and the $95 \%$ confidence intervals were calculated. The difference between the three patient groups was assessed using a Kruskal-Wallis analysis of variance. A rank sum test was employed to show the recovery of $\mathrm{PGI}_{2}$ production by HUVEC and to express the results from the 6-keto $\mathrm{PGF}_{1 \alpha}$ assay.

\section{Results}

\section{PGI $_{2}$ SYNTHESIS BY HUVEC}

The sera from 12 normal adults induced $\mathrm{PGI}_{2}$ synthesis by HUVEC (fig 1), as measured by inhibition of platelet aggregation by culture supernatants (median percentage full aggregation $21.5 ; 95 \%$ confidence interval 7 to 36 ). In contrast, the serum from 10 of 13 patients with meningococcal shock failed to support endothelial $\mathrm{PGI}_{2}$ production (median percentage full aggregation $89 ; 95 \%$ confidence interval 36 to 98). Similar $\mathrm{PGI}_{2}$ stimulating activity to the controls was observed with the sera from 16 of

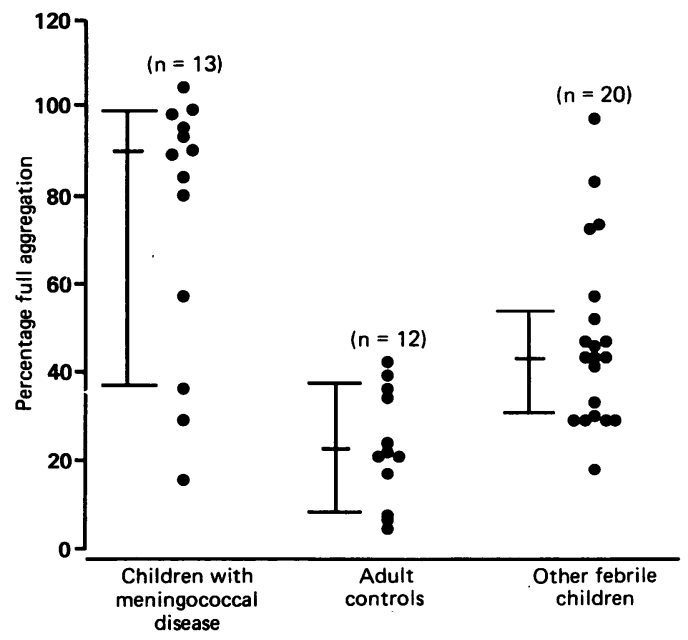

Figure 1 Human umbilical vein endothelial cell $P G I_{2}$ production after incubation with sera from patients with meningococcal shock, adult controls, and children with other febrile illnesses (Kruskal-Wallis analysis of variance, $p<0.05$ ) 
20 children with other febrile illnesses (median percentage full aggregation $43 ; 95 \%$ confidence interval 30 to 52). In four children, three with severe illness (pneumococcal meningitis, toxic shock syndrome, and bacteroides septicaemia respectively) and one with Lyme disease, $\mathrm{PGI}_{2}$ production was diminished. The three patient groups were shown to be different by KruskalWallis analysis of variance $(p<0.05)$.

The difference between the sera from the patients with meningococcal shock and the adult controls demonstrated by bioassay was confirmed using the 6-keto $\mathrm{PGF}_{1 \alpha}$ radioimmunoassay. On incubation of HUVEC with sera from meningococcal shock patients, the median 6-keto $\mathrm{PGF}_{1 \alpha}$ detected was $4 \cdot 2 \mathrm{ng} / \mathrm{ml}$ (range $2 \cdot 7-6 \cdot 6$ ) in comparison to $11.3 \mathrm{ng} / \mathrm{ml}$ (range 2.2-31) with HUVEC incubated with control sera (rank sum test, $\mathrm{p}<0.03$ ).

\section{CROSSOVER EXPERIMENTS}

When HUVEC previously cultured with sera from patients with meningococcal shock were reincubated with normal serum there was a significant increase in $\mathrm{PGI}_{2}$ production (rank sum test, $\mathrm{p}<0.03$ ) (fig 2).

\section{CHARACTERISATION OF THE DEFECT}

Heating sera from patients with meningococcal shock to $56^{\circ} \mathrm{C}$ for either 10 or 30 minutes did not affect its activity. Storage of these sera for more than two weeks at $4^{\circ} \mathrm{C}$ did not change their effect on $\mathrm{PGI}_{2}$ synthesis. Mixing experiments, employing the bioassay, have shown that addition of as little as one part normal serum to three parts serum from patients with meningococcal shock, restores the ability to induce endothelial $\mathrm{PGI}_{2}$ production (results not shown), suggesting that normal serum is augmenting the meningococcal serum rather than reversing an inhibitor.

\section{PLATELET AND CLOTTING STUDIES}

Platelet and clotting studies were available from

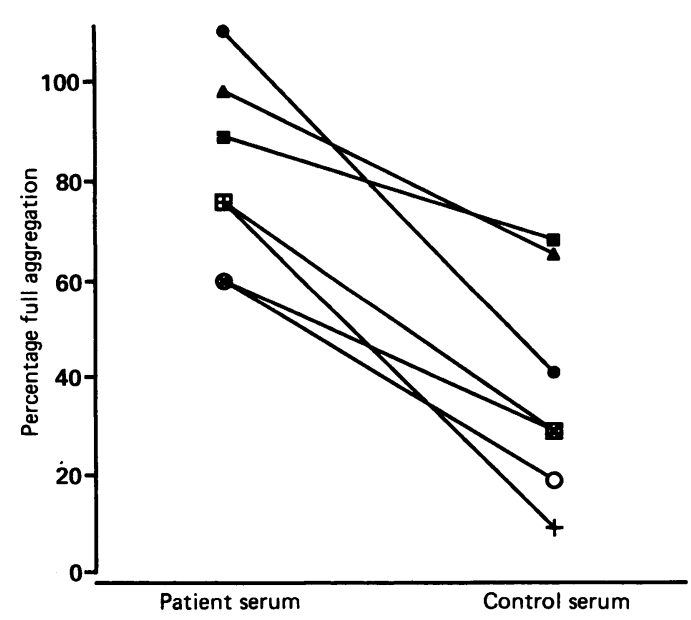

Figure 2 Crossover experiments. Human umbilical vein endothelial cells initially cultured with the sera from meningococcal patients and then reincubated with adult control sera recovered the ability to produce $P G I_{2}$ (rank sum test, $p<0.03$ ).

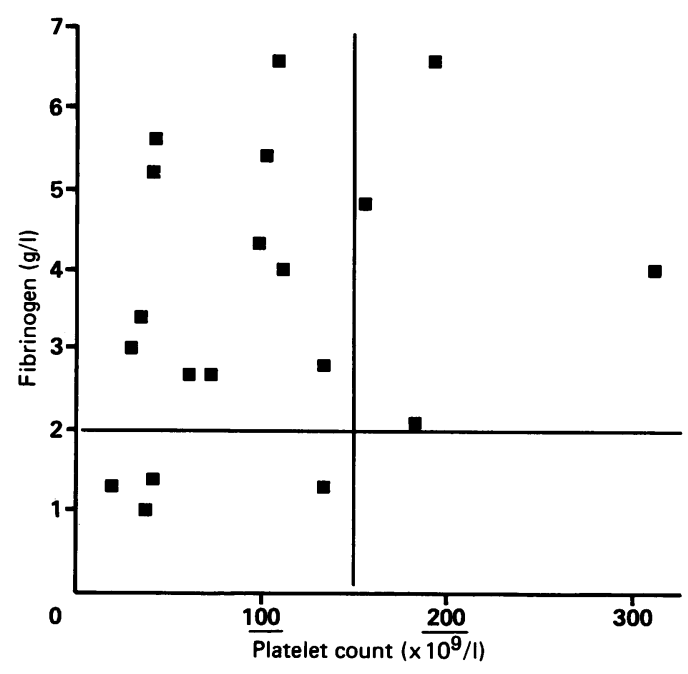

Figure 3 The platelet counts plotted against the fibrinogen concentrations from 19 children with severe meningococcal disease measured in the first 24 hours of the disease (bars indicate the lower limits of normal).

the records of 19 of the 23 children with severe meningococcal disease. In the first 24 hours after admission, $79 \%$ of these patients were thrombocytopenic but only $21 \%$ had low fibrinogen concentrations (fig 3). Platelet aggregation studies were performed on two patients with meningococcal shock. A poor response to ADP and adrenalin was observed, implying that in vivo platelet activation had occurred.

\section{Discussion}

We have shown a reduction in $\mathrm{PGI}_{2}$ synthesis by HUVEC when incubated with the sera from 10 of 13 children with meningococcal shock when compared with normal controls. The abnormality was demonstrated both by the standard bioassay for $\mathrm{PGI}_{2}$ and by the measurement of 6-keto $\mathrm{PGF}_{1 \alpha}$. The effect of sera from children with meningococcal shock on HUVEC was shown to be reversible, establishing that the $\mathrm{PGI}_{2}$ deficiency was not due to a permanent alteration in endothelial cell function. The defect was not affected by heating to $56^{\circ} \mathrm{C}$ or by prolonged storage in the cold. Mixing experiments suggest that this phenomenon may be due to the absence of a factor necessary for $\mathrm{PGI}_{2}$ synthesis rather than an inhibitor. The defect is not specific for meningococcal shock as it was also observed with the sera from four of 20 children with other febrile illnesses. The clinical manifestations and pathophysiology of severe infections overlap and it is therefore not surprising that similar defects may occur in the late stages of a variety of infections. None the less, if these in vitro findings reflect the in vivo situation, patients with meningococcal shock may have a defect in $\mathrm{PGI}_{2}$ production by the vascular endothelium. This may be an important factor in the vasoconstriction, intravascular thrombosis, and platelet consumption seen in the disorder.

Impaired $\mathrm{PGI}_{2}$ synthesis has been implicated in the pathogenesis of a number of other diseases, including sporadic haemolytic uraemic 
syndrome, ${ }^{11}$ systemic lupus erythematosis, ${ }^{6}$ and pre-eclamptic toxaemia. ${ }^{6}$ These conditions are all associated with endothelial damage, platelet activation, and the formation of microthrombi. We therefore looked for features of platelet activation and disseminated intravascular coagulation in 19 children with severe meningococcal sepsis in the first $\mathbf{2 4}$ hours of the illness. Whereas fibrinogen concentrations were normal or increased, thrombocytopenia was present in the majority of patients and was often profound and persistent. The impaired response to platelet agonists documented in the small number of patients studied by ourselves and others 5 suggest that in meningococcal disease platelets circulate in an 'exhausted' form. ${ }^{12}$ Animal studies have also demonstrated intravascular platelet activation in endotoxaemia, ${ }^{13}$ which together with the observed failure of heparin treatment to reduce the intravascular thrombosis of meningococcaemia, ${ }^{5}$ adds weight to the possibility that platelet activation and consumption may be more important than the depletion of coagulation factors in this disease.

If endothelial $\mathrm{PGI}_{2}$ synthesis is diminished in meningococcal disease, this may be an important area for therapeutic intervention. In animal models, $\mathrm{PGI}_{2}$ infusions have been shown to be effective in reversing the consequences of endotoxaemia, improving renal perfusion, and ameliorating intravascular thrombosis. ${ }^{13} 14$ Bihari and Tinker have found $\mathrm{PGI}_{2}$ to be a valuable vasodilator in critically ill adults, improving oxygen delivery and so tissue oxygen consumption. Further studies to confirm the presence of defective $\mathrm{PGI}_{2}$ production in vivo and to evaluate the potential use of exogenous $\mathrm{PGI}_{2}$ in meningococcal shock are needed.
We would like to thank Ms J Cookson for her help and advice in the platelet work, Ms A Wade for her statistical advice, and Dr M Michaels for helping to collect the patient data. We would also like to thank Dr DJ Bihari and Dr I Murdock for their support. RSH is a Medical Research Council training fellow.

1 Havens PL, Garland JS, Brook MM, Dewitz BA, Stremsk ES, Troshynski TJ. Trends in mortality in children hospitalised with meningococcal infections, 1957 to 1987. Pospitalised with meningococcal

2 Mercier J-C, Beaufils F, Hartman J-F, Azéma D. Hemodynamic patterns of meningococcal shock in children. Crit ed 1988;16:27-33.

3 Levin M. Shock. In: Black D, ed. Paediatric emergencies. 2nd Ed. London: Butterworths, 1987:87-116.

4 McGehee WG, Rapaport SI, Hjort PF. Intravascular coagulation in fulminant meningococcemia. Ann Intern Med 1967;67:250-60

5 Gérard P, Moriau M, Bachy A, Malvaux P, De Meyer R Meningococcal purpura: report of 19 patients treated with heparin. I Pediatr 1973;82:780-6.

6 Moncada S. Biological importance of prostacyclin. $\mathrm{Br} f$ Pharmacol 1982;76:3-31.

7 Radomski MW, Palmer RMJ, Moncada S. The anti-aggregating properties of vascular endothelium: interactions between prostacyclin and nitric oxide. Br $\mathcal{F}$ Pharmacol 1987;92:639-46.

8 Busse R, Förstermann, Matsuda H, Pohl U. The role of prostaglandins in the endothelium-mediated vasodilatory response to hypoxia. Pflugers Arch 1984;401:77-83.

9 Gimbrone MA, Cotran RS, Folkman J. Human vascular endothelium in culture: growth and DNA synthesis. $7 \mathrm{Cel}$ Biol 1974;60:673-84

10 Moncada S, Gryglewski R, Bunting S, Vane JR. An enzyme isolated from arteries transforms prostaglandin endoperoxides to an unstable substance that inhibits platele aggregation. Nature 1976;263:663-5.

11 Levin M, Elkon KB, Nokes TJC, et al. Inhibitor of prostacyclin production in sporadic haemolytic uraemic syndrome. Arch Dis Child 1983;58:703-8.

12 Paretti FI, Capitanio, Mannucci L, Ponticelli C, Mannucci PM. Acquired dysfunction due to the circulation of 'exhausted' platelets. Am F Med 1980:69:235-9.

13 Campos A, Kim Y, Azar SH, Vernier RL, Michael AF Prevention of the generalised Shwartzman reaction in pregnant rats by prostacyclin infusion. Lab Invest 1983;48: pregnant

14 Ditter H, Matthias FR, Voss R, Lohmann E. Beneficial effects prostacyclin in a rabbit endotoxin shock model. effects prostacyclin in a rab
Thromb Res 1988;51:403-15.

15 Bihari DJ, Tinker J. The therapeutic value of vasodilator prostaglandins in multiple organ failure associated with sepsis. Intensive Care Medicine 1988;15:3-7. 